International Journal of Pure and Applied Mathematics

Volume 86 No. $1 \quad$ 2013, 217-234

ISSN: 1311-8080 (printed version); ISSN: 1314-3395 (on-line version)

url: http://www.ijpam.eu

doi: http://dx.doi.org/10.12732/ijpam.v86i1.14

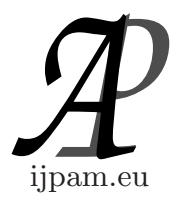

\title{
DIRICHLET PROBLEM FOR ELLIPTIC EQUATIONS IN WEIGHTED SOBOLEV SPACES
}

\author{
Serena Boccia $^{1}$, Sara Monsurrò ${ }^{2}$, Maria Transirico ${ }^{3}$ \\ 1,2,3 Departement of Mathematics \\ University of Salerno \\ Via Giovanni Paolo II, 132 Fisciano (SA), I-84084, ITALY
}

\begin{abstract}
We study the Dirichlet problem for uniformly elliptic second order linear differential equations with discontinuous coefficients in an unbounded domain of $\mathbb{R}^{n}, n \geq 2$, in weighted Sobolev spaces. An a priori bound and an existence and uniqueness result are proved.
\end{abstract}

AMS Subject Classification: 35J25, 35B45, 35R05

Key Words: elliptic equations, discontinuous coefficients, a priori bounds, weighted Sobolev spaces

\section{Introduction}

The suitability of weighted Sobolev spaces in the theory of PDEs with prescribed boundary conditions is well known. In particular, they find a natural field of application in the case of unbounded domains, thanks to the introduction of an opportune weight function that controls the behaviour of the solution at infinity.

Received: May 29, 2013

(C) 2013 Academic Publications, Ltd. url: www.acadpubl.eu

${ }^{\S}$ Correspondence author 
In this framework, given an unbounded open subset $\Omega$ of $\mathbb{R}^{n}, n \geq 2$, we study the uniformly elliptic second order linear differential operator with discontinuous coefficients

$$
L=-\sum_{i, j=1}^{n} a_{i j} \frac{\partial^{2}}{\partial x_{i} \partial x_{j}}+\sum_{i=1}^{n} a_{i} \frac{\partial}{\partial x_{i}}+a
$$

and the related Dirichlet problem

$$
\left\{\begin{array}{l}
u \in W_{s}^{2, p}(\Omega) \cap \stackrel{\circ}{W}_{s}^{1, p}(\Omega), \\
L u=f, \quad f \in L_{s}^{p}(\Omega),
\end{array}\right.
$$

where $p>1, s \in \mathbb{R}$, and $W_{s}^{2, p}(\Omega), \stackrel{\circ}{W}_{s}^{1, p}(\Omega)$ and $L_{s}^{p}(\Omega)$ are some classes of weighted Sobolev and Lebesgue spaces recently introduced in [18], where a complete account of their properties can be found. In these spaces, the weight $\rho^{s}$ is a power of a function $\rho$ of class $C^{2}(\bar{\Omega})$ such that $\rho: \bar{\Omega} \rightarrow \mathbb{R}_{+}$and

$$
\begin{gathered}
\sup _{x \in \Omega} \frac{\left|\partial^{\alpha} \rho(x)\right|}{\rho(x)}<+\infty \quad \forall|\alpha| \leq 2, \\
\lim _{|x| \rightarrow+\infty}\left(\rho(x)+\frac{1}{\rho(x)}\right)=+\infty \quad \text { and } \lim _{|x| \rightarrow+\infty} \frac{\rho_{x}(x)+\rho_{x x}(x)}{\rho(x)}=0 .
\end{gathered}
$$

As an example, one can think of the function

$$
\rho(x)=\left(1+|x|^{2}\right)^{t}, \quad t \in \mathbb{R} \backslash\{0\} .
$$

Concerning the hypotheses on the coefficients of the operator $L$, we consider the very general case that the $a_{i j}$ are bounded, are locally VMO, and are "close" at infinity to certain functions $e_{i j}$ having vanishing mean oscillation, that is a kind of continuity in the average sense and not in the pointwise sense. As for the lower-order terms coefficients we suppose that they verify suitable regularity hypotheses and have a prescribed behaviour at the infinity.

Our main result is the following weighted a priori estimate:

$$
\|u\|_{W_{s}^{2, p}(\Omega)} \leq c\left(\|L u\|_{L_{s}^{p}(\Omega)}+\|u\|_{L^{p}\left(\Omega_{1}\right)}\right) \quad \forall u \in W_{s}^{2, p}(\Omega) \cap \stackrel{\circ}{W_{s}^{1, p}(\Omega),}
$$

where $c \in \mathbb{R}_{+}$is a constant whose dependence is fully described and $\Omega_{1}$ is a bounded open subset of $\Omega$. As an application of this a priori bound, an existence and uniqueness theorem for the solution of problem (1.1) has been established. 
If $n \geq 3$, the no-weighted problem corresponding to (1.1) has been studied by several authors under various hypotheses on the $a_{i j}$. We quote here the first existence and uniqueness theorem regarding VMO coefficients, obtained in $[13,14]$, assuming that $\Omega$ is bounded, the $a_{i j} \in L^{\infty}(\Omega) \cap V M O(\Omega)$ and $a_{i}=a=$ 0 . This latter condition has been dropped later on in $[30,31]$. Recently, these results have been extended to the case of unbounded open sets in no-weighted contests (see [8,9] for $n \geq 3$, and [12] for $n=2$ ) and also in weighted ones (see $[2,3,4,5,6])$. Variational problems can be found in $[7,16,17,19,20,21]$ and related weighted and no-weighted results are proved in [22, 23, 24].

\section{Weight Functions and Weighted Spaces}

This section is devoted to a class of weighted Sobolev spaces recently introduced in [18], where all the details concerning the properties stated below can be found. Once the definition is given, we recall a fundamental lemma allowing to use no-weighted results in order to pass to the weighted contest. Finally, we remind some auxiliary functions, somehow related to the weight and satisfying opportune properties, needed throughout this work.

From now on let $\Omega$ be an open subset of $\mathbb{R}^{n}, n \geq 2$, not necessarily bounded, and consider a weight function $\rho: \bar{\Omega} \rightarrow \mathbb{R}_{+}$such that $\rho \in C^{2}(\bar{\Omega})$ and

$$
\sup _{x \in \Omega} \frac{\left|\partial^{\alpha} \rho(x)\right|}{\rho(x)}<+\infty \quad \forall|\alpha| \leq 2 .
$$

To fix the ideas, one can think of $\rho$ as the function

$$
\rho(x)=\left(1+|x|^{2}\right)^{t}, \quad t \in \mathbb{R} .
$$

For $k \in \mathbb{N}_{0}, p \in[1,+\infty[$ and $s \in \mathbb{R}$, and given a weight $\rho$ satisfying (2.1), we define $W_{s}^{k, p}(\Omega)$ as the space of distributions $u$ on $\Omega$ such that

$$
\|u\|_{W_{s}^{k, p}(\Omega)}=\sum_{|\alpha| \leq k}\left\|\rho^{s} \partial^{\alpha} u\right\|_{L^{p}(\Omega)}<+\infty
$$

endowed with the norm in (2.2). Furthermore, we denote the closure of $C_{\circ}^{\infty}(\Omega)$ in $W_{s}^{k, p}(\Omega)$ by $\stackrel{\circ}{W}_{s}^{k, p}(\Omega)$ and put $W_{s}^{0, p}(\Omega)=L_{s}^{p}(\Omega)$.

One of the most important characteristics of the above mentioned weighted spaces is contained in the following lemma: 
Lemma 2.1. Let $k \in \mathbb{N}_{0}, p \in[1,+\infty[$ and $s \in \mathbb{R}$. If $\Omega$ has the segment property, then the map

$$
u \longrightarrow \rho^{s} u
$$

defines a topological isomorphism from $W_{s}^{k, p}(\Omega)$ to $W^{k, p}(\Omega)$ and from $\stackrel{\circ}{W_{s}^{k, p}}(\Omega)$ to $\stackrel{\circ}{W}^{k, p}(\Omega)$.

In the sequel the additional assumptions below are also needed

$$
\lim _{|x| \rightarrow+\infty}\left(\rho(x)+\frac{1}{\rho(x)}\right)=+\infty \quad \text { and } \quad \lim _{|x| \rightarrow+\infty} \frac{\rho_{x}(x)+\rho_{x x}(x)}{\rho(x)}=0 .
$$

As an example, we can therefore consider

$$
\rho(x)=\left(1+|x|^{2}\right)^{t}, \quad t \in \mathbb{R} \backslash\{0\} .
$$

To our purpose it is essential to associate to the weight $\rho$ the function $\sigma$ defined by

$$
\left\{\begin{array}{lll}
\sigma=\rho & \text { if } \rho \rightarrow+\infty & \text { for }|x| \rightarrow+\infty \\
\sigma=\frac{1}{\rho} & \text { if } \rho \rightarrow 0 & \text { for }|x| \rightarrow+\infty .
\end{array}\right.
$$

It is easily seen that $\sigma$ verifies (2.1) too, and moreover

$$
\lim _{|x| \rightarrow+\infty} \sigma(x)=+\infty, \quad \lim _{|x| \rightarrow+\infty} \frac{\sigma_{x}(x)+\sigma_{x x}(x)}{\sigma(x)}=0 .
$$

Now, fix a cutoff function $f \in C_{\circ}^{\infty}\left(\overline{\mathbb{R}}_{+}\right)$such that

$$
0 \leq f \leq 1, \quad f(t)=1 \text { if } t \in[0,1], \quad f(t)=0 \text { if } t \in[2,+\infty[
$$

and set

$$
\zeta_{k}: x \in \bar{\Omega} \longrightarrow f\left(\frac{\sigma(x)}{k}\right), \quad k \in \mathbb{N}
$$

and

$$
\Omega_{k}=\{x \in \Omega: \sigma(x)<k\}, \quad k \in \mathbb{N} .
$$

One has that, introduced the sequence

$$
\eta_{k}: x \in \bar{\Omega} \longrightarrow 2 k \zeta_{k}(x)+\left(1-\zeta_{k}(x)\right) \sigma(x), \quad k \in \mathbb{N},
$$

for any $k \in \mathbb{N}$

$$
\sigma \sim \eta_{k}
$$


Furthermore, for any $k \in \mathbb{N}$,

$$
\begin{array}{ll}
\frac{\left(\eta_{k}\right)_{x}}{\eta_{k}} \leq c_{1}^{\prime} \sup _{\overline{\Omega \backslash \Omega}} \frac{\sigma_{x}}{\sigma} & \text { in } \bar{\Omega} \\
\frac{\left(\eta_{k}\right)_{x x}}{\eta_{k}} \leq c_{2}^{\prime} \sup _{\bar{\Omega}_{k}} \frac{\sigma_{x}^{2}+\sigma \sigma_{x x}}{\sigma^{2}} & \text { in } \bar{\Omega}
\end{array}
$$

and moreover

$$
\lim _{k \rightarrow+\infty} \frac{\sup }{\Omega \backslash \Omega_{k}} \frac{\sigma_{x}(x)+\sigma_{x x}(x)}{\sigma(x)}=0 .
$$

\section{The Spaces of the Coefficients}

Here we recall the definitions and the main properties of the functional spaces where the coefficients of our operator belong.

Let us give some notation. Let $G$ be any Lebesgue measurable subset of $\mathbb{R}^{n}$ and $\Sigma(G)$ the collection of all Lebesgue measurable subsets of $G$. For $F \in \Sigma(G)$, $|F|$ denotes its Lebesgue measure, $\chi_{F}$ its characteristic function and $\mathrm{D}(F)$ the class of restrictions to $F$ of functions $\zeta \in C_{\circ}^{\infty}\left(\mathbb{R}^{n}\right)$ with $\bar{F} \cap \operatorname{supp} \zeta \subseteq F$. If $X(F)$ is a space of functions defined on $F, X_{\text {loc }}(F)$ denotes the class of all functions $g: F \rightarrow \mathbb{R}$ such that $\zeta g \in X(F)$ for any $\zeta \in \mathrm{D}(F)$. Finally, for any $x \in \mathbb{R}^{n}$ and $r \in \mathbb{R}_{+}$, we put $B(x, r)=\left\{y \in \mathbb{R}^{n}:|y-x|<r\right\}, B_{r}=B(0, r)$ and $F(x, r)=F \cap B(x, r)$.

In the sequel let us assume that $\Omega$ is an unbounded open subset of $\mathbb{R}^{n}$, $n \geq 2$.

If $\Omega$ is such that

$$
\left.\left.|\Omega(x, r)| \geq A r^{n} \quad \forall x \in \Omega, \quad \forall r \in\right] 0,1\right],
$$

where $A$ is a positive constant independent of $x$ and $r$, then we can consider the space $B M O(\Omega, \tau)\left(\tau \in \mathbb{R}_{+}\right)$of functions $g \in L_{\text {loc }}^{1}(\bar{\Omega})$ such that

$$
[g]_{B M O(\Omega, \tau)}=\sup _{\substack{x \in \Omega \\ r \in[0, \tau]}} f_{\Omega(x, r)}\left|g-f_{\Omega(x, r)} g\right|<+\infty,
$$

with

$$
f_{\Omega(x, r)} g=|\Omega(x, r)|^{-1} \int_{\Omega(x, r)} g .
$$


If $g \in B M O(\Omega)=B M O\left(\Omega, \tau_{A}\right)$, and

$$
\tau_{A}=\sup \left\{\tau \in \mathbb{R}_{+}: \sup _{\substack{x \in \Omega \\ r \in] 0, \tau]}} \frac{r^{n}}{|\Omega(x, r)|} \leq \frac{1}{A}\right\}
$$

we say that $g \in V M O(\Omega)$ if $[g]_{B M O(\Omega, \tau)} \rightarrow 0$ for $\tau \rightarrow 0^{+}$.

For more details on VMO functions we refer the reader to [25], where they were defined for the first time, and to [29] for details in the case of unbounded domains.

A function

$$
\eta[g]:] 0,1] \longrightarrow \mathbb{R}_{+}
$$

is called a modulus of continuity of $g$ in $V M O(\Omega)$ if

$$
\left.\left.[g]_{B M O(\Omega, \tau)} \leq \eta[g](\tau) \forall \tau \in\right] 0,1\right], \quad \lim _{\tau \rightarrow 0^{+}} \eta[g](\tau)=0
$$

For $\lambda \in\left[0, n\left[, q \in\left[1,+\infty\left[\right.\right.\right.\right.$, the space of Morrey type $M^{q, \lambda}(\Omega, \tau)\left(\tau \in \mathbb{R}_{+}\right)$ is the set of all functions $g$ in $L_{l o c}^{q}(\bar{\Omega})$ such that

$$
\|g\|_{M^{q, \lambda}(\Omega, \tau)}=\sup _{\substack{r \in] 0, \tau] \\ x \in \Omega}} r^{-\lambda / q}\|g\|_{L^{q}(\Omega(x, r))}<+\infty
$$

endowed with the norm defined in (3.2). These spaces generalize to unbounded domains the classical notion of Morrey spaces and were introduced in [28] (see also [10], for further characterizations).

Since for any $\tau_{1}, \tau_{2} \in \mathbb{R}_{+}$, a function $g$ belongs to $M^{q, \lambda}\left(\Omega, \tau_{1}\right)$ if and only if it belongs to $M^{q, \lambda}\left(\Omega, \tau_{2}\right)$ and the norms of $g$ in these two spaces are equivalent, we can restrict our attention to the space $M^{q, \lambda}(\Omega)=M^{q, \lambda}(\Omega, 1)$.

We recall that one has

$$
M^{q, \lambda}(\Omega) \subseteq M^{q_{0}, \lambda_{0}}(\Omega) \quad \text { if } q_{0} \leq q \text { and } \frac{\lambda_{0}-n}{q_{0}} \leq \frac{\lambda-n}{q} .
$$

We denote by $M_{\circ}^{q, \lambda}(\Omega)$ the closure of $C_{\circ}^{\infty}(\Omega)$ in $M^{q, \lambda}(\Omega)$ and put $M^{q}(\Omega)=$ $M^{q, 0}(\Omega)$ and $M_{\circ}^{q}(\Omega)=M_{\circ}^{q, 0}(\Omega)$.

For reader's convenience let us mention that, as observed in [27], for $q \in$ $[1,+\infty[$, one has

$$
f \in L_{l o c}^{q}(\bar{\Omega}), \quad \lim _{|x| \rightarrow+\infty} f(x)=0 \Rightarrow f \in M_{\circ}^{q}(\Omega) .
$$


In order to define the modulus of continuity of functions belonging to $M_{\circ}^{q, \lambda}(\Omega)$, for $h \in \mathbb{R}_{+}$and $g \in M^{q, \lambda}(\Omega)$, we set

$$
F[g](h)=\sup _{\substack{E \in \Sigma(\Omega) \\ \sup _{x \in \Omega}|E(x, 1)| \leq \frac{1}{\hbar}}}\left\|g \chi_{E}\right\|_{M^{q, \lambda}(\Omega)} .
$$

Then, we recall that given a function $g \in M^{q, \lambda}(\Omega)$ the following characterization holds:

$$
g \in M_{\circ}^{q, \lambda}(\Omega) \Longleftrightarrow \lim _{h \rightarrow+\infty}\left(F[g](h)+\left\|\left(1-\zeta_{h}\right) g\right\|_{M^{q, \lambda}(\Omega)}\right)=0,
$$

where $\zeta_{h}$ denotes a function of class $C_{o}^{\infty}\left(\mathbb{R}^{n}\right)$ such that

$$
0 \leq \zeta_{h} \leq 1, \quad \zeta_{\left.h\right|_{B(0, h)}}=1, \quad \operatorname{supp} \zeta_{h} \subset B(0,2 h) .
$$

Thus, if $g$ is a function in $M_{\circ}^{q, \lambda}(\Omega)$ a modulus of continuity of $g$ in $M_{\circ}^{q, \lambda}(\Omega)$ is an application $\sigma_{o}^{q, \lambda}[g]: \mathbb{R}_{+} \rightarrow \mathbb{R}_{+}$such that

$$
\begin{gathered}
F[g](h)+\left\|\left(1-\zeta_{h}\right) g\right\|_{M^{q, \lambda}(\Omega)} \leq \sigma_{o}^{q, \lambda}[g](h), \\
\lim _{h \rightarrow+\infty} \sigma_{o}^{q, \lambda}[g](h)=0 .
\end{gathered}
$$

In the sequel a modulus of continuity of a function $g$ in $M_{\circ}^{q}(\Omega)$ will be denoted by $\sigma_{o}^{q}[g]$.

Theorem 3.1 of [15], adapted to our framework, guarantees the following embedding result:

Theorem 3.1. Let $p>1$ and assume that $k$ and $t$ are numbers such that:

$$
k \in \mathbb{N}, t \geq p, t \geq \frac{n}{k}, \text { with } t>p \text { if } p=\frac{n}{k} .
$$

If $\Omega$ has the cone property and $g \in M^{t}(\Omega)$, then for any $u \in W_{s}^{k, p}(\Omega)$ we have $g u \in L_{s}^{p}(\Omega)$ and

$$
\|g u\|_{L_{s}^{p}(\Omega)} \leq c\|g\|_{M^{t}(\Omega)}\|u\|_{W_{s}^{k, p}(\Omega)},
$$

with $c$ positive constant depending only on $\Omega, n, k, p$ and $t$.

As a straightforward consequence, we can prove the corollary below:

Corollary 3.2. Let $p>1$ and assume that $k$ and $t$ are numbers such that: $k \in \mathbb{N}, t \geq p, t \geq \frac{n}{k}$, with $t>p$ if $p=\frac{n}{k}$. 
If $\Omega$ has the cone property and $g \in M_{\circ}^{t}(\Omega)$, then for any $\varepsilon \in \mathbb{R}_{+}$there exist a positive constant $c(\varepsilon) \in \mathbb{R}_{+}$and a bounded open subset $\Omega_{\varepsilon} \subset \subset \Omega$ with the cone property such that

$$
\|g u\|_{L_{s}^{p}(\Omega)} \leq \varepsilon\|u\|_{W_{s}^{k, p}(\Omega)}+c(\varepsilon)\|u\|_{L^{p}\left(\Omega_{\varepsilon}\right)} \forall u \in W_{s}^{k, p}(\Omega),
$$

with $c(\varepsilon)$ and $\Omega_{\varepsilon}$ depending only on $\varepsilon, \Omega, n, k, p, \rho, s, t, \sigma_{\circ}^{t}[g]$.

Proof. Fix $\varepsilon>0$ and let $c$ be the constant in (3.4). Since $g \in M_{\circ}^{t}(\Omega)$, there exists $g_{\varepsilon} \in C_{\circ}^{\infty}(\Omega)$ such that $\left\|g-g_{\varepsilon}\right\|_{M^{t}(\Omega)}<\frac{\varepsilon}{c}$. Let $\Omega_{\varepsilon}$ be a bounded open subset of $\Omega$, with the cone property, such that supp $g_{\varepsilon} \subset \Omega_{\varepsilon}$, by Theorem 3.1 and taking into account that $\rho \in C^{2}(\bar{\Omega})$ one has

$$
\begin{aligned}
\|g u\|_{L_{s}^{p}(\Omega)} & \leq c\left\|g-g_{\varepsilon}\right\|_{M^{t}(\Omega)}\|u\|_{W_{s}^{k, p}(\Omega)}+\left\|g_{\varepsilon} u\right\|_{L_{s}^{p}\left(\Omega_{\varepsilon}\right)} \\
& \leq \varepsilon\|u\|_{W_{s}^{k, p}(\Omega)}+\left\|g_{\varepsilon} \rho^{s}\right\|_{L^{\infty}\left(\Omega_{\varepsilon}\right)}\|u\|_{L^{p}\left(\Omega_{\varepsilon}\right)}
\end{aligned}
$$

for any $u$ in $W_{s}^{k, p}(\Omega)$. This concludes the proof.

\section{An a Priori Bound}

From now on, let $p>1$ and $s \in \mathbb{R}$ and suppose that

$\left(h_{0}\right) \quad \Omega$ is an unbounded open subset of $\mathbb{R}^{n}, n \geq 2$, with the uniform $C^{1,1}$-regularity property.

We consider in $\Omega$ the differential operator

$$
L=-\sum_{i, j=1}^{n} a_{i j} \frac{\partial^{2}}{\partial x_{i} \partial x_{j}}+\sum_{i=1}^{n} a_{i} \frac{\partial}{\partial x_{i}}+a,
$$

with the following conditions on the leading coefficients:

$\left(h_{1}\right) \quad\left\{\begin{array}{l}a_{i j}=a_{j i} \in L^{\infty}(\Omega) \cap V M O_{l o c}(\bar{\Omega}), \quad i, j=1, \ldots, n, \\ \exists \nu>0: \quad \sum_{i, j=1}^{n} a_{i j} \xi_{i} \xi_{j} \geq \nu|\xi|^{2} \quad \text { a.e. in } \Omega, \forall \xi \in \mathbb{R}^{n} .\end{array}\right.$

Furthermore, assume that there exist some functions $e_{i j}, i, j=1, \ldots, n$, 
and $g$, and a positive real constant $\mu$ such that

$\left(h_{2}\right) \quad\left\{\begin{array}{l}\sum_{i, j=1}^{n} e_{i j} \xi_{i} \xi_{j} \geq \mu|\xi|^{2} \quad \text { a.e. in } \Omega, \quad \forall \xi \in \mathbb{R}^{n}, \\ \lim _{r \rightarrow+\infty} \sum_{i, j=1}^{n}\left\|e_{i j}-g a_{i j}\right\|_{L^{\infty}\left(\Omega \backslash B_{r}\right)}=0 \\ \text { with } g \in L^{\infty}(\Omega), g_{0}=\underset{\Omega}{\operatorname{ess}} \inf g>0\end{array}\right.$

For the lower order term coefficients assume that

$\left(h_{3}\right) \quad\left\{\begin{array}{l}a_{i} \in M_{\circ}^{t_{1}}(\Omega), \quad i=1, \ldots, n, \\ a=a^{\prime}+a^{\prime \prime}, a^{\prime} \in M_{\circ}^{t_{2}}(\Omega), a^{\prime \prime} \in L^{\infty}(\Omega), a_{0}^{\prime \prime}=\underset{\Omega}{\operatorname{ess} \inf a^{\prime \prime}>0,} \\ \text { with } t_{1}=t \text { and } t_{2}=p \text { for } n=2, \\ \text { and with } t_{1} \geq p, \quad t_{1} \geq n, \quad t_{1}>p \quad \text { if } p=n, \\ t_{2} \geq p, \quad t_{2} \geq n / 2, \quad t_{2}>p \quad \text { if } p=n / 2 \quad \text { for } n>2 .\end{array}\right.$

We remark that under assumptions $\left(h_{0}\right)-\left(h_{3}\right)$, in view of Theorem 3.1, the operator $L: W_{s}^{2, p}(\Omega) \rightarrow L_{s}^{p}(\Omega)$ is bounded.

Set

$$
L_{0}=-\sum_{i, j=1}^{n} a_{i j} \frac{\partial^{2}}{\partial x_{i} \partial x_{j}},
$$

the following preliminary bound holds true:

Lemma 4.1. Suppose that assumptions $\left(h_{0}\right)-\left(h_{2}\right)$ hold and that $a^{\prime \prime}$ is as in $\left(h_{3}\right)$. Then, there are two real positive constants $c$ and $r_{0}$ and a bounded open set $\Omega_{0} \subset \subset \Omega$, with the cone property, such that

$$
\|u\|_{W^{2, p}(\Omega)} \leq c\left(\left\|\left(L_{0}+a^{\prime \prime}\right) u\right\|_{L^{p}(\Omega)}+\|u\|_{L^{p}\left(\Omega_{0}\right)}\right) \quad \forall u \in W^{2, p}(\Omega) \cap \stackrel{\circ}{W^{1, p}}(\Omega),
$$

where $c$ and $\Omega_{0}$ depend on $n, p, \Omega, \nu, \mu, g_{0}, a_{0}^{\prime \prime}, t,\left\|a_{i j}\right\|_{L^{\infty}(\Omega)},\left\|e_{i j}\right\|_{L^{\infty}(\Omega)}$, $\|g\|_{L^{\infty}(\Omega)},\left\|a^{\prime \prime}\right\|_{L^{\infty}(\Omega)}, \eta\left[\zeta_{2 r_{0}} a_{i j}\right], \sigma_{0}^{t, \lambda}\left[\left(e_{i j}\right)_{x}\right]$ and $r_{0}$ depends on $n, p, \Omega, \mu, g_{0}$, $a_{0}^{\prime \prime}, t,\left\|e_{i j}\right\|_{L^{\infty}(\Omega)},\|g\|_{L^{\infty}(\Omega)},\left\|a^{\prime \prime}\right\|_{L^{\infty}(\Omega)}, \sigma_{0}^{t, \lambda}\left[\left(e_{i j}\right)_{x}\right]$. 
Proof. For $n \geq 3$ the thesis can be obtained applying Theorem 3.3 of [9] to the operator $L_{0}+a^{\prime \prime}$.

For $n=2$ one can proceed as in the proof of Theorem 3.3 of [9] replacing the applications of Theorem 5.1 of [11] with those of Lemma 3.1 of [12].

Now we can prove our main a priori estimate.

Theorem 4.2. Suppose that assumptions $\left(h_{0}\right)-\left(h_{3}\right)$ hold and that the weight function $\rho$ satisfies (2.3). Then, there are two real positive constants $c$ and $r_{0}$ and a bounded open set $\Omega_{1} \subset \subset \Omega$, with the cone property, such that

$$
\|u\|_{W_{s}^{2, p}(\Omega)} \leq c\left(\|L u\|_{L_{s}^{p}(\Omega)}+\|u\|_{L^{p}\left(\Omega_{1}\right)}\right) \quad \forall u \in W_{s}^{2, p}(\Omega) \cap \stackrel{\circ}{W_{s}^{1, p}}(\Omega),
$$

where $c$ and $\Omega_{1}$ depend on $n, p, \Omega, \nu, \mu, g_{0}, a_{0}^{\prime \prime}, t, t_{1}, t_{2}, \rho, s,\left\|a_{i j}\right\|_{L^{\infty}(\Omega)}$, $\left\|e_{i j}\right\|_{L^{\infty}(\Omega)},\|g\|_{L^{\infty}(\Omega)},\left\|a^{\prime \prime}\right\|_{L^{\infty}(\Omega)}, \eta\left[\zeta_{2 r_{0}} a_{i j}\right], \sigma_{0}^{t, \lambda}\left[\left(e_{i j}\right)_{x}\right], \sigma_{0}^{t_{1}}\left[a_{i}\right], \sigma_{0}^{t_{2}}\left[a^{\prime}\right]$ and $r_{0}$ depends on $n, p, \Omega, \mu, g_{0}, a_{0}^{\prime \prime}, t,\left\|e_{i j}\right\|_{L^{\infty}(\Omega)},\|g\|_{L^{\infty}(\Omega)},\left\|a^{\prime \prime}\right\|_{L^{\infty}(\Omega)}, \sigma_{0}^{t, \lambda}\left[\left(e_{i j}\right)_{x}\right]$.

Proof. Let $u \in W_{s}^{2, p}(\Omega) \cap \stackrel{\circ}{W_{s}^{1, p}}(\Omega)$. In view of Lemma 2.1

$$
\rho^{s} u \in W^{2, p}(\Omega) \cap \stackrel{\circ}{W^{1, p}}(\Omega) .
$$

Therefore, applying Lemma 4.1 we have that there exist two real numbers $c_{0}, r_{0} \in \mathbb{R}_{+}$and an open bounded subset $\Omega_{0} \subset \subset \Omega$ with the cone property such that

$$
\left\|\rho^{s} u\right\|_{W^{2, p}(\Omega)} \leq c_{0}\left(\left\|\left(L_{0}+a^{\prime \prime}\right)\left(\rho^{s} u\right)\right\|_{L^{p}(\Omega)}+\left\|\rho^{s} u\right\|_{L^{p}\left(\Omega_{0}\right)}\right),
$$

where $c_{0}$ and $\Omega_{0}$ depend on $n, p, \Omega, \nu, \mu, g_{0}, a_{0}^{\prime \prime}, t,\left\|a_{i j}\right\|_{L^{\infty}(\Omega)},\left\|e_{i j}\right\|_{L^{\infty}(\Omega)},\|g\|_{L^{\infty}(\Omega)}$, $\left\|a^{\prime \prime}\right\|_{L^{\infty}(\Omega)}, \eta\left[\zeta_{2 r_{0}} a_{i j}\right], \sigma_{0}^{t, \lambda}\left[\left(e_{i j}\right)_{x}\right]$, and $r_{0}$ depends on $n, p, \Omega, \mu, g_{0}, a_{0}^{\prime \prime}, \mathrm{t},\left\|e_{i j}\right\|_{L^{\infty}(\Omega)}$, $\|g\|_{L^{\infty}(\Omega)},\left\|a^{\prime \prime}\right\|_{L^{\infty}(\Omega)}, \sigma_{0}^{t, \lambda}\left[\left(e_{i j}\right)_{x}\right]$.

If we observe that

$$
\begin{gathered}
\left(L_{0}+a^{\prime \prime}\right)\left(\rho^{s} u\right)=\rho^{s} L u-s(s-1) \rho^{s-2} \sum_{i, j=1}^{n} a_{i j} \rho_{x_{i}} \rho_{x_{j}} u- \\
2 s \rho^{s-1} \sum_{i, j=1}^{n} a_{i j} \rho_{x_{i}} u_{x_{j}}-s \rho^{s-1} \sum_{i, j=1}^{n} a_{i j} \rho_{x_{i} x_{j}} u-\rho^{s} \sum_{i=1}^{n} a_{i} u_{x_{i}}-\rho^{s} a^{\prime} u
\end{gathered}
$$

we obtain

$$
\|u\|_{W_{s}^{2, p}(\Omega)} \leq c_{1}\left(\|L u\|_{L_{s}^{p}(\Omega)}+\|u\|_{L^{p}\left(\Omega_{0}\right)}+\sum_{i, j=1}^{n}\left\|\rho^{s-2} \rho_{x_{i}} \rho_{x_{j}} u\right\|_{L^{p}(\Omega)}+\right.
$$




$$
\begin{aligned}
& \sum_{i, j=1}^{n}\left\|\rho^{s-1} \rho_{x_{i}} u_{x_{j}}\right\|_{L^{p}(\Omega)}+\sum_{i, j=1}^{n}\left\|\rho^{s-1} \rho_{x_{i} x_{j}} u\right\|_{L^{p}(\Omega)}+ \\
& \left.\sum_{i=1}^{n}\left\|a_{i} u_{x_{i}}\right\|_{L_{s}^{p}(\Omega)}+\left\|a^{\prime} u\right\|_{L_{s}^{p}(\Omega)}\right)
\end{aligned}
$$

where $c_{1}$ depends on the same parameters as $c_{0}$ and on $\rho, s$.

The regularity of $\rho$ and the second hypothesis in (2.3) put us in a position to exploit (3.3) in order to deduce from Corollary 3.2 that for any $\varepsilon \in \mathbb{R}_{+}$ and $i, j=1, \ldots, n$ there exist $c_{1}(\varepsilon), c_{2}(\varepsilon), c_{3}(\varepsilon) \in \mathbb{R}_{+}$and some bounded open subsets $\Omega_{1}(\varepsilon) \subset \subset \Omega, \Omega_{2}(\varepsilon) \subset \subset \Omega, \Omega_{3}(\varepsilon) \subset \subset \Omega$ with the cone property such that

$$
\begin{aligned}
& \left\|\rho^{-2} \rho_{x_{i}} \rho_{x_{j}} u\right\|_{L_{s}^{p}(\Omega)} \leq \varepsilon\|u\|_{W_{s}^{2, p}(\Omega)}+c_{1}(\varepsilon)\|u\|_{L^{p}\left(\Omega_{1}(\varepsilon)\right)}, \\
& \left\|\rho^{-1} \rho_{x_{i}} u_{x_{j}}\right\|_{L_{s}^{p}(\Omega)} \leq \varepsilon\|u\|_{W_{s}^{2, p}(\Omega)}+c_{2}(\varepsilon)\left\|u_{x_{j}}\right\|_{L^{p}\left(\Omega_{2}(\varepsilon)\right)}, \\
& \left\|\rho^{-1} \rho_{x_{i} x_{j}} u\right\|_{L_{s}^{p}(\Omega)} \leq \varepsilon\|u\|_{W_{s}^{2, p}(\Omega)}+c_{3}(\varepsilon)\|u\|_{L^{p}\left(\Omega_{3}(\varepsilon)\right)},
\end{aligned}
$$

where $c_{1}(\varepsilon), c_{2}(\varepsilon), c_{3}(\varepsilon), \Omega_{1}(\varepsilon), \Omega_{2}(\varepsilon), \Omega_{3}(\varepsilon)$ are dependent on $\varepsilon, \Omega, n, p, \rho, s$.

Moreover, using again Corollary 3.2 and Theorem 4.7 of [1] we have that there exist $c_{4}(\varepsilon), c_{5}(\varepsilon) \in \mathbb{R}_{+}$and two bounded open sets $\Omega_{4}(\varepsilon) \subset \subset \Omega, \Omega_{5}(\varepsilon) \subset \subset$ $\Omega$ with the cone property such that:

$$
\begin{gathered}
\left\|a_{i} u_{x_{i}}\right\|_{L_{s}^{p}(\Omega)} \leq \varepsilon\|u\|_{W_{s}^{2, p}(\Omega)}+c_{4}(\varepsilon)\left\|u_{x_{i}}\right\|_{L^{p}\left(\Omega_{4}(\varepsilon)\right)} \leq \\
\varepsilon\|u\|_{W_{s}^{2, p}(\Omega)}+c_{4}(\varepsilon)\left(\left\|u_{x x}\right\|_{L^{p}\left(\Omega_{4}(\varepsilon)\right)}^{\frac{1}{2}}\|u\|_{L^{p}\left(\Omega_{4}(\varepsilon)\right)}^{\frac{1}{2}}+\|u\|_{L^{p}\left(\Omega_{4}(\varepsilon)\right)}\right), \\
\left\|a^{\prime} u\right\|_{L_{s}^{p}(\Omega)} \leq \varepsilon\|u\|_{W_{s}^{2, p}(\Omega)}+c_{5}(\varepsilon)\|u\|_{L^{p}\left(\Omega_{5}(\varepsilon)\right)},
\end{gathered}
$$

where $c_{4}(\varepsilon)$ and $\Omega_{4}(\varepsilon)$ depend on $\varepsilon, \Omega, n, p, \rho, s, t_{1}, \sigma_{0}^{t_{1}}\left[a_{i}\right]$ and $c_{5}(\varepsilon)$ and $\Omega_{5}(\varepsilon)$ depend on $\varepsilon, \Omega, n, p, \rho, s, t_{2}, \sigma_{0}^{t_{2}}\left[a^{\prime}\right]$.

From (4.2)-(4.7) we have

$$
\begin{gathered}
\|u\|_{W_{s}^{2, p}(\Omega)} \leq c_{2}\left(\|L u\|_{L_{s}^{p}(\Omega)}+\|u\|_{L_{p}\left(\Omega_{0}\right)}+\right. \\
\varepsilon\|u\|_{W_{s}^{2, p}(\Omega)}+c_{6}(\varepsilon)\left(\left\|u_{x x}\right\|_{L^{p}\left(\Omega_{6}(\varepsilon)\right)}^{\frac{1}{2}}\|u\|_{L^{p}\left(\Omega_{6}(\varepsilon)\right)}^{\frac{1}{2}}+\|u\|_{\left.\left.L^{p}\left(\Omega_{6}(\varepsilon)\right)\right)\right),}\right.
\end{gathered}
$$

where $c_{2}$ depends on the same parameters as $c_{1}, c_{6}(\varepsilon)$ is the maximum between the constants $c_{1}(\varepsilon), c_{2}(\varepsilon), c_{3}(\varepsilon), c_{4}(\varepsilon), c_{5}(\varepsilon)$, and $\Omega_{6}(\varepsilon)$ is a bounded open subset 
of $\Omega$ with the cone property such that $\Omega_{6}(\varepsilon) \subset \subset \Omega$ and $\Omega_{6}(\varepsilon) \supset \Omega_{1}(\varepsilon) \cup \Omega_{2}(\varepsilon) \cup$ $\Omega_{3}(\varepsilon) \cup \Omega_{4}(\varepsilon) \cup \Omega_{5}(\varepsilon)$. If we choose $\varepsilon=\frac{1}{2 c_{2}}$, from the last inequality we deduce

$$
\begin{aligned}
& \|u\|_{W_{s}^{2, p}(\Omega)} \leq c_{3}\left(\|L u\|_{L_{s}^{p}(\Omega)}+\|u\|_{L_{p}\left(\Omega_{0}\right)}+\right. \\
& \left.\left.\left\|u_{x x}\right\|_{L^{p}\left(\Omega_{6}(\varepsilon)\right)}^{\frac{1}{2}}\|u\|_{L^{p}\left(\Omega_{6}(\varepsilon)\right)}^{\frac{1}{2}}+\|u\|_{L^{p}\left(\Omega_{6}(\varepsilon)\right)}\right)\right),
\end{aligned}
$$

where $c_{3}$ depends on the same parameters as $c_{2}$ and on $t_{1}, t_{2}, \sigma_{0}^{t_{1}}\left[a_{i}\right], \sigma_{0}^{t_{2}}\left[a^{\prime}\right]$. Now, using Young's inequality, (4.8) can be rewritten as

$$
\begin{gathered}
\|u\|_{W_{s}^{2, p}(\Omega)} \leq c_{3}\left(\|L u\|_{L_{s}^{p}(\Omega)}+\|u\|_{L_{p}\left(\Omega_{0}\right)}+\right. \\
\left.\left.\varepsilon^{\prime}\left\|u_{x x}\right\|_{L^{p}\left(\Omega_{6}(\varepsilon)\right)}+c\left(\varepsilon^{\prime}\right)\|u\|_{L^{p}\left(\Omega_{6}(\varepsilon)\right)}+\|u\|_{L^{p}\left(\Omega_{6}(\varepsilon)\right)}\right)\right),
\end{gathered}
$$

where $c\left(\varepsilon^{\prime}\right)$ is a positive constant depending only on $\varepsilon^{\prime}$.

From (4.9), choosing $\varepsilon^{\prime}=\frac{1}{2 c_{3}}$ and denoting by $\Omega_{1}$ a bounded open subset of $\Omega$ with the cone property such that $\Omega_{1} \subset \subset \Omega$ and $\Omega_{1} \supset \Omega_{6}(\varepsilon) \cup \Omega_{0}$, we have the result.

\section{Uniqueness and Existence Results}

In this section we want to obtain an existence and uniqueness result for a Dirichlet problem associated to the operator (4.1). A preliminary result, whose proof can be found in [23], is needed.

Lemma 5.1. If hypothesis $\left(h_{0}\right)$ is satisfied, then the Dirichlet problem

$$
\left\{\begin{array}{l}
u \in W_{s}^{2, p}(\Omega) \cap \stackrel{\circ}{W}_{s}^{1, p}(\Omega), \\
-\Delta u+b u=f, \quad f \in L_{s}^{p}(\Omega),
\end{array}\right.
$$

is uniquely solvable, with

$$
b=1+\left|-s(s+1) \sum_{i=1}^{n} \frac{\sigma_{x_{i}}^{2}}{\sigma^{2}}+s \sum_{i=1}^{n} \frac{\sigma_{x_{i} x_{i}}}{\sigma}\right|,
$$

if $\rho \rightarrow+\infty$ for $|x| \rightarrow+\infty$, or

$$
b=1+\left|-s(s-1) \sum_{i=1}^{n} \frac{\sigma_{x_{i}}^{2}}{\sigma^{2}}-s \sum_{i=1}^{n} \frac{\sigma_{x_{i} x_{i}}}{\sigma}\right|,
$$

if $\rho \rightarrow 0$ for $|x| \rightarrow+\infty$. 
To obtain the solvability of the Dirichlet problem we are interested in stronger assumptions are required:

$\left(h_{3}^{\prime}\right) \quad\left\{\begin{array}{l}a_{i} \in M_{\circ}^{t_{1}}(\Omega), i=1, \ldots, n, \\ a=a^{\prime}+a^{\prime \prime}, a^{\prime} \in M_{\circ}^{t_{2}}(\Omega), a^{\prime \prime} \in L^{\infty}(\Omega), a_{0}^{\prime \prime}=\underset{\Omega}{\operatorname{ess} \inf } a^{\prime \prime}>0, \\ \text { with } t_{1}=t \text { and } t_{2}=p \text { for } n=2, \\ \text { and with } t_{1}>n \text { if } p \leq n, \quad t_{1}=p \text { if } p>n, \\ t_{2}>n / 2 \text { if } p \leq n / 2, \quad t_{2}=p \text { if } p>n / 2 \text { for } n>2, \\ g \in \operatorname{Lip}(\bar{\Omega}) .\end{array}\right.$

Theorem 5.2. Suppose that assumptions $\left(h_{0}\right)-\left(h_{2}\right)$ and $\left(h_{3}^{\prime}\right)$ hold and that the weight function $\rho$ satisfies (2.3). Then the problem

$$
\left\{\begin{array}{l}
u \in W_{s}^{2, p}(\Omega) \cap \stackrel{\circ}{W_{s}^{1, p}}(\Omega), \\
L u=0,
\end{array}\right.
$$

admits only the zero solution.

Proof. Fix $u \in W_{s}^{2, p}(\Omega) \cap \stackrel{\circ}{W_{s}^{1, p}}(\Omega)$. If $\rho \rightarrow+\infty$ for $|x| \rightarrow+\infty, \sigma=\rho$, hence by Lemma 2.1 one has that $\sigma^{s} u \in W^{2, p}(\Omega) \cap \stackrel{\circ}{W^{1, p}}(\Omega)$.

Thus, if we write, $\eta_{k}=\eta$, for a fixed $k \in \mathbb{N}$, since $\eta$ and $\sigma$ are equivalent, one also has that $\eta^{s} u \in W^{2, p}(\Omega) \cap \stackrel{\stackrel{\circ}{W}}{1, p}(\Omega)$. Therefore, Theorem 5.2 of [12], for $n=2$, and Theorem 4.3 of [9], for $n \geq 3$, together with the bounded inverse theorem (see Theorem 3.8 in [26]) give that there exists $c_{0} \in \mathbb{R}_{+}$, independent of $u$ and $k$, such that

$$
\left\|\eta^{s} u\right\|_{W^{2, p}(\Omega)} \leq c_{0}\left\|L\left(\eta^{s} u\right)\right\|_{L^{p}(\Omega)} .
$$

Simple computations give then

$$
\begin{aligned}
L\left(\eta^{s} u\right)= & \eta^{s} L u-s \sum_{i, j=1}^{n} a_{i j}\left((s-1) \eta^{s-2} \eta_{x_{i}} \eta_{x_{j}} u+\eta^{s-1} \eta_{x_{i} x_{j}} u+\right. \\
& \left.2 \eta^{s-1} \eta_{x_{i}} u_{x_{j}}\right)+s \sum_{i=1}^{n} a_{i} \eta^{s-1} \eta_{x_{i}} u .
\end{aligned}
$$


Using (5.5) and (5.6) we deduce that

$$
\begin{aligned}
\left\|\eta^{s} u\right\|_{W^{2, p}(\Omega)} \leq & c_{1}\left(\left\|\eta^{s} L u\right\|_{L^{p}(\Omega)}+\sum_{i, j=1}^{n}\left(\left\|\eta^{s-2} \eta_{x_{i}} \eta_{x_{j}} u\right\|_{L^{p}(\Omega)}+\right.\right. \\
& \left.\left\|\eta^{s-1} \eta_{x_{i} x_{j}} u\right\|_{L^{p}(\Omega)}+\left\|\eta^{s-1} \eta_{x_{i}} u_{x_{j}}\right\|_{L^{p}(\Omega)}\right)+ \\
& \left.\sum_{i=1}^{n}\left\|a_{i} \eta^{s-1} \eta_{x_{i}} u\right\|_{L^{p}(\Omega)}\right)
\end{aligned}
$$

with $c_{1}$ independent of $u$ and $k$.

On the other hand, from Theorem 3.1 and (2.5) we get

$$
\left\|a_{i} \eta^{s-1} \eta_{x_{i}} u\right\|_{L^{p}(\Omega)} \leq c_{2} \frac{\sup }{\Omega \backslash \Omega_{k}} \frac{\sigma_{x}}{\sigma}\left\|a_{i}\right\|_{M^{t_{1}(\Omega)}}\left\|\eta^{s} u\right\|_{W^{1, p}(\Omega)},
$$

with $c_{2}$ independent of $u$ and $k$.

Putting together (2.5), (2.6), (5.7) and (5.8), we obtain the bound

$$
\begin{aligned}
\left\|\eta^{s} u\right\|_{W^{2, p}(\Omega)} \leq & c_{3}\left[\left\|\eta^{s} L u\right\|_{L^{p}(\Omega)}+\left(\sup _{\Omega \backslash \Omega_{k}} \frac{\sigma_{x}^{2}+\sigma \sigma_{x x}}{\sigma^{2}}+\right.\right. \\
& \left.\left.\frac{\sup }{\Omega \backslash \Omega_{k}} \frac{\sigma_{x}}{\sigma}\right)\left\|\eta^{s} u\right\|_{W^{2, p}(\Omega)}\right]
\end{aligned}
$$

with $c_{3}$ independent of $u$ and $k$.

Observe that by $(2.7)$ it follows that there exists $k_{O} \in \mathbb{N}$ such that

$$
\left(\sup _{\overline{\Omega \backslash \Omega_{k_{o}}}} \frac{\sigma_{x}^{2}+\sigma \sigma_{x x}}{\sigma^{2}}+\frac{\sup }{\Omega \backslash \Omega_{k_{o}}} \frac{\sigma_{x}}{\sigma}\right) \leq \frac{1}{2 c_{3}} .
$$

Therefore, if we still denote by $\eta$ the function $\eta_{k_{o}}$, combining (5.9) and (5.10) we obtain

$$
\left\|\eta^{s} u\right\|_{W^{2, p}(\Omega)} \leq 2 c_{3}\left\|\eta^{s} L u\right\|_{L^{p}(\Omega)} .
$$

This, together with (2.4) written for $k=k_{o}$ and Lemma 2.1 applied considering $\sigma$ as weight function, gives

$$
\sum_{|\alpha| \leq 2}\left\|\sigma^{s} \partial^{\alpha} u\right\|_{L^{p}(\Omega)} \leq c_{4}\left\|\sigma^{s} L u\right\|_{L^{p}(\Omega)}
$$

with $c_{4}$ independent of $u$. This means that

$$
\|u\|_{W_{s}^{2, p}(\Omega)} \leq c\|L u\|_{L_{s}^{p}(\Omega)}, \quad \forall u \in W_{s}^{2, p}(\Omega) \cap \stackrel{\circ}{W}_{s}^{1, p}(\Omega),
$$


with $c$ independent of $u$. Thus, problem (5.4) admits only the zero solution.

If $\rho \rightarrow 0$ for $|x| \rightarrow+\infty$, then $\sigma=\rho^{-1}$, thus, always in view of the isomorphism of Lemma 2.1, one has that $\sigma^{-s} u \in W^{2, p}(\Omega) \cap \stackrel{\circ}{W^{1, p}}(\Omega)$. Therefore arguing as to get (5.11) one obtains

$$
\sum_{|\alpha| \leq 2}\left\|\sigma^{-s} \partial^{\alpha} u\right\|_{L^{p}(\Omega)} \leq c_{5}\left\|\sigma^{-s} L u\right\|_{L^{p}(\Omega)} .
$$

This concludes the proof of Theorem 5.2.

We are now in a position to establish the solvability of our weighted problem by means of the following existence and uniqueness result:

Theorem 5.3. Suppose that assumptions $\left(h_{0}\right)-\left(h_{2}\right)$ and $\left(h_{3}^{\prime}\right)$ hold and that the weight function $\rho$ satisfies (2.3). Then the problem

$$
\left\{\begin{array}{l}
u \in W_{s}^{2, p}(\Omega) \cap \stackrel{\circ}{W_{s}^{1, p}}(\Omega) \\
L u=f, \quad f \in L_{s}^{p}(\Omega),
\end{array}\right.
$$

is uniquely solvable.

Proof. For each $\tau \in[0,1]$ we put

$$
L_{\tau}=\tau L+(1-\tau)(-\Delta+b),
$$

with $b$ defined in (5.2) if $\rho \rightarrow+\infty$ for $|x| \rightarrow+\infty$, or in (5.3) if $\rho \rightarrow 0$ for $|x| \rightarrow+\infty$.

Let us start observing that the function

$$
\tau \in[0,1] \longmapsto L_{\tau} \in B\left(W_{s}^{2, p}(\Omega) \cap \stackrel{\circ}{W_{s}^{1, p}}(\Omega), L_{s}^{p}(\Omega)\right)
$$

is continuous, in view of Theorem 3.1. Moreover, by Theorem 4.2 one has that the operator $L_{\tau}$ has closed range and by Theorem 5.2 it also has null kernel. Thus, Lemma 4.1 of [9] applies giving that there exists a positive real number c such that

$$
\begin{array}{r}
\|u\|_{W_{s}^{2, p}(\Omega)} \leq c|| L_{\tau} u \|_{L_{s}^{p}(\Omega)} \\
\forall u \in W_{s}^{2, p}(\Omega) \cap \stackrel{\circ}{W}_{s}^{1, p}(\Omega), \quad \forall \tau \in[0,1] .
\end{array}
$$

Furthermore, by Lemma 5.1 problem (5.1) is uniquely solvable. Putting together this latter result and estimate (5.14) and using the method of continuity along a parameter, we finally get that problem (5.13) is uniquely solvable too. 


\section{References}

[1] S. Boccia, L. Caso, Interpolation inequalities in weighted Sobolev spaces, J. Math. Inequal., 2 (2008), 309-322, doi: org/10.7153/jmi-02-28.

[2] S. Boccia, S. Monsurrò, M. Transirico, Elliptic equations in weighted Sobolev spaces on unbounded domains, Int. J. Math. Math. Sci., vol. 2008 (2008), ID 582435, 12 pages, doi: 10.1155/2008/582435.

[3] S. Boccia, S. Monsurrò, M. Transirico, Solvability of the Dirichlet problem for elliptic equations in weighted Sobolev spaces on unbounded domains, Bound. Value Probl., vol. 2008 (2008), ID 901503, 13 pages, doi: 10.1155/2008/901503.

[4] S. Boccia, M. Salvato, M. Transirico, A priori bounds for elliptic operators in weighted Sobolev spaces, J. Math. Inequal., 6 (2012), 307-318, doi: 10.7153/jmi-06-31.

[5] S. Boccia, M. Salvato, M. Transirico, Existence and uniqueness results for Dirichlet problem in weighted Sobolev spaces on unbounded domains, Methods Appl. Anal., 18 (2011), 203-214.

[6] S. Boccia, M. Salvato, M. Transirico, The Dirichlet problem for elliptic equations in weighted Sobolev spaces on unbounded domains of the plane, Math. Slovaca, to appear.

[7] G. Bottaro, M.E. Marina, Problema di Dirichlet per equazioni ellittiche di tipo variazionale su insiemi non limitati, Boll. Un. Mat. Ital., 8 (1973), 46-56.

[8] L. Caso, P. Cavaliere, M. Transirico, Uniqueness results for elliptic equations with VMO - coefficients, Int. J. Pure Appl. Math., 13 (2004), 499-512.

[9] L. Caso, P. Cavaliere, M. Transirico, An existence result for elliptic equations with VMO-coefficients, J. Math. Anal. Appl., 325 (2007), 10951102 .

[10] L. Caso, R. D'Ambrosio, S. Monsurrò, Some remarks on spaces of Morrey type, Abstr. Appl. Anal., vol. 2010 (2010), 22 pages, doi: $10.1155 / 2010 / 242079$.

[11] P. Cavaliere, M. Longobardi, A. Vitolo, Imbedding estimates and elliptic equations with discontinuous coefficients in unbounded domains, Matematiche (Catania), 51 (1996), 87-104. 
[12] P. Cavaliere, M. Transirico, The Dirichlet problem for elliptic equations in unbounded domains of the plane, J. Funct. Spaces Appl., 8 (2008), 47-58, doi: $10.1155 / 2008 / 872891$.

[13] F. Chiarenza, M. Frasca, P. Longo, Interior $W^{2, p}$ estimates for non divergence elliptic equations with discontinuous coefficients, Ricerche Mat., 40 (1991), 149-168.

[14] F. Chiarenza, M. Frasca, P. Longo, $W^{2, p}$-solvability of the Dirichlet problem for nondivergence elliptic equations with VMO coefficients, Trans. Amer. Math. Soc., 336 (1993), 841-853.

[15] A.V. Glushak - M. Transirico - M. Troisi, Teoremi di immersione ed equazioni ellittiche in aperti non limitati, Rend. Mat., 9 (1989), 113-130.

[16] P.L. Lions, Remarques sur les équations linéaires elliptiques du second ordre sous forme divergence dans les domaines non bornés, Atti Accad. Naz. Lincei Rend. Cl. Sci. Fis. Mat. Natur., 78 (1985), 205-212.

[17] P.L. Lions, Remarques sur les équations linéaires elliptiques du second ordre sous forme divergence dans les domaines non bornés II, Atti Accad. Naz. Lincei Rend. Cl. Sci. Fis. Mat. Natur., 79 (1985), 178-183.

[18] S. Monsurrò, M. Salvato, M. Transirico, $W^{2,2}$-a priori bounds for a class of elliptic operators, Int. J. Differ. Equ., vol. 2011 (2011), 17 pages, doi: $10.1155 / 2011 / 572824$.

[19] S. Monsurrò, M. Transirico, A $L^{p}$-estimate for weak solutions of elliptic equations, Abstr. Appl. Anal., vol. 2012 (2012), 15 pages, doi: $10.1155 / 2012 / 376179$.

[20] S. Monsurrò, M. Transirico, Dirichlet problem for divergence form elliptic equations with discontinuous coefficients, Bound. Value Probl., vol. 2012 (2012), 20 pages, doi: 10.1186/1687-2770-2012-67.

[21] S. Monsurrò, M. Transirico, A priori bounds in $L^{p}$ for solutions of elliptic equations in divergence form, Bull. Sci. Math. (2013), doi: 10.1016/j.bulsci.2013.02.002.

[22] S. Monsurrò, M. Transirico, A $W^{2, p}$-estimate for a class of elliptic operators, Int. J. Pure Appl. Math., 83 (2013), 489-499, doi: 10.12732/ijpam.v83i3.9. 
[23] S. Monsurrò, M. Transirico, A Weighted $W^{2, p}$-bound for a class of elliptic operators, J. Inequal. Appl., 263 (2013), doi: 10.1186/1029-242X-2013263.

[24] S. Monsurrò, M. Transirico, A priori bounds in $L^{p}$ and in $W^{2, p}$ for solutions of elliptic equations, Abstr. Appl. Anal. (2013), in press.

[25] D. Sarason, Functions of vanishing mean oscillation, Trans. Amer. Math. Soc., 207 (1975), 391-405.

[26] M. Schechter, Principles of Functional Analysis, American Mathematical Society, Providence, RI, (2002).

[27] M. Transirico, M. Troisi, Equazioni ellittiche del secondo ordine di tipo non variazionale in aperti non limitati, Ann. Mat. Pura Appl., 152 (1988), 209-226.

[28] M. Transirico, M. Troisi, A. Vitolo, Spaces of Morrey type and elliptic equations in divergence form on unbounded domains, Boll. Un. Mat. Ital., 9 B (1995), 153-174.

[29] M. Transirico, M. Troisi, A. Vitolo, BMO spaces on domains of $\mathbb{R}^{n}$, Ricerche Mat., 45 (1996), 355-378.

[30] C. Vitanza, $W^{2, p}$-regularity for a class of elliptic second order equations with discontinuous coefficients, Matematiche (Catania), 47 (1992), 177 186.

[31] C. Vitanza, A new contribution to the $W^{2, p}$-regularity for a class of elliptic second order equations with discontinuous coefficients, Matematiche (Catania), 48 (1993), 287-296. 